\title{
Enamel Hypoplaisa in a Young Female Patient
}

\author{
Apeksha Ghai ${ }^{*}$, Mayur Kaushik ${ }^{2}$, Nitin Tomar $^{3}$ and Amit Wadhawan ${ }^{3}$ \\ ${ }^{1}$ Post-Graduate Student, Subharti Dental College, Department of Periodontology, India \\ ${ }^{2}$ Head of Department, Department of Periodontology, Subharti Dental College, India \\ ${ }^{3}$ Proffessor, Department of Periodontology, Subharti Dental College, India \\ *Corresponding Author: Apeksha Ghai, Department of Periodontology, Subharti Dental College, India.
}

Received: June 14, 2019; Published: July 08, 2019

DOI: $10.31080 /$ ASDS.2019.03.0585

\begin{abstract}
Enamel hypoplasia is a defect of the tooth surface of a crown caused by disturbance of enamel matrix secretion. Enamel defects can be congenital or acquired.. Development defects of enamel (DDE) comprise of mainly dental defects such as hypoplasia and diffuse and demarcated opacities; fluorosis and amelogenesis imperfecta. Enamel hypoplasia, thus, is a surface defect of the tooth crown that is caused by a disturbance of enamel matrix secretion, defective calcification or defective maturation.
\end{abstract}

Keywords: Enamel; Hypoplasia; Mottle; Amelogenesis; Defects

\section{Introduction}

Ameloblasts are the cells that produce the enamel .Their life cycle is divided into six stages that include morphogenetic, organizing, formative, maturative, protective, and desmolytic. Amelogenesis occurs during the formative and maturative stages of the ameloblasts. Enamel matrix is secreted in the formative stage where as mineralization of the enamel matrix occurs in the maturation stage. The principal expressions of pathologic amelogenesis are the hypoplasia, hypocalcification or hypomineralization. Enamel hypoplasia occurs if matrix formation is affected and may manifest as pitting, grooving or even total absence of enamel. Hypomineralization results when maturation is disturbed and is manifested in the form of opaque or chalky areas on normally contoured enamel surfaces. Thus, disturbance either in matrix formation or in calcification can occur depending chiefly on the stage of tooth formation at the time of injury [1].

Dental defects such as hypoplasia and diffuse and demarcated opacities; fluorosis and amelogenesis imperfect are classified as DEE (Developmental Defects of enamel). Enamel hypoplasia, thus, is a surface defect of the tooth crown that is caused by a disturbance of enamel matrix secretion, defective calcification or defective maturation.2 Enamel hypoplasia or hypo mineralization may be caused by hereditary factors and environmental factors that include systemic factors such as nutritional factors, exanthematous diseases like measles and chicken pox, congenital syphilis, hypocalcemia, birth injury or premature birth, fluoride ingestion or idiopathic causes, and local factors such as infection or trauma from a deciduous tooth.

Hereditary enamel hypoplasia/hypomineralization is known as amelogenesis imperfecta. Familial transmission is seen as a mendelian dominant trait which affects enamel of all the teeth, deciduous as well as permanent. Environmental enamel hypoplasia/hypomineralization of systemic or local origin is also termed as "chronologic hypoplasia". This lesion is found in areas of those teeth where the enamel was being formed during the systemic or local disturbance. Since the formation of enamel extends over a long period and the systemic or local disturbance, in most cases are of short duration, the defect is limited to a circumscribed area of the affected teeth or tooth. Thus knowing the chronologic development of deciduous and permanent teeth will make it possible to determine from the location of the defect, the approximate time at which the injury occurred $[2,3]$.

The basic pathology in DDE is the result of abrupt, shortterm or long-term ameloblastic insults during the secretory or maturation phase of the development of tooth; any systemic illness, disturbance, deficiency or prematurity of neonate or local trauma can lead to DDE [3]. Enamel hypoplasia can also be seen in 
other pediatric conditions in which hypocalcemia is a major sign as in rickets, prematurity and neonatal tetany [4]. Furthermore, disturbances in the development of the enamel of permanent teeth can result from trauma to the primary teeth because of the close proximity of the root of the primary teeth to their permanent successors [5]. Enamel hypoplasia may be inherited or may result from an illness, malnutrition, trauma or due to fluorosis [6]. Although it can occur in any permanent tooth, the most commonly involved sites of hypoplasia are the permanent first molars and incisors with specific areas of defect and well-demarcated areas of hypomineralization [7]. The secretory phase for development of the permanent incisors and first molars begins in utero whereas the maturation process begins at birth and thus any trauma of inadequate mineralization can result in DDE [8-10].

Within the first year of birth, teeth have formed enamel hypoplasia. The most affected teeth are first permanent molars and incisor. Mixed areas of hypoplasia and hypomineralisation are clinically.

\section{Case Report}

A 36 year-old female patient visited the Department of Periodontology at the Subharti Dental College and Hospital with chief complaint of malodour and stained teeth. On giving the past dental history, it was discovered that, this was patients first visit to a dental hospital. Patient reported that brown staining with mottled appearance (Figure 1) on her teeth were present in her deciduous dentition as well. A yellowish brown appearance of enamel was noted on first clinical examination. Medical history revealed no systemic conditions or any parafunctional habits.

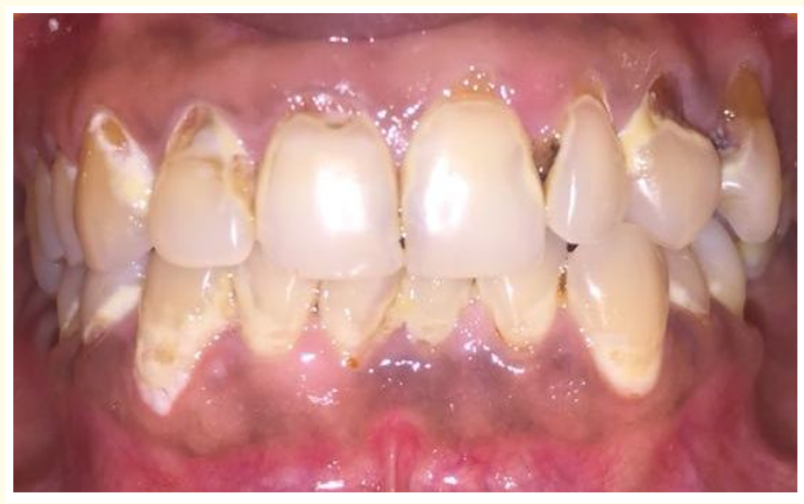

Figure 1
The patient was apparently well and was suggested to get scaling done for the removal of tartar. While performing the non-surgical therapy, the operator noticed small flakes of enamel being chipped out upon the use of the ultrasonic scaler (Figure 2). On carefully examining the patient, it was discovered that the patient suffered from Enamel Hypoplasia or Amelogenesis Imperfecta.

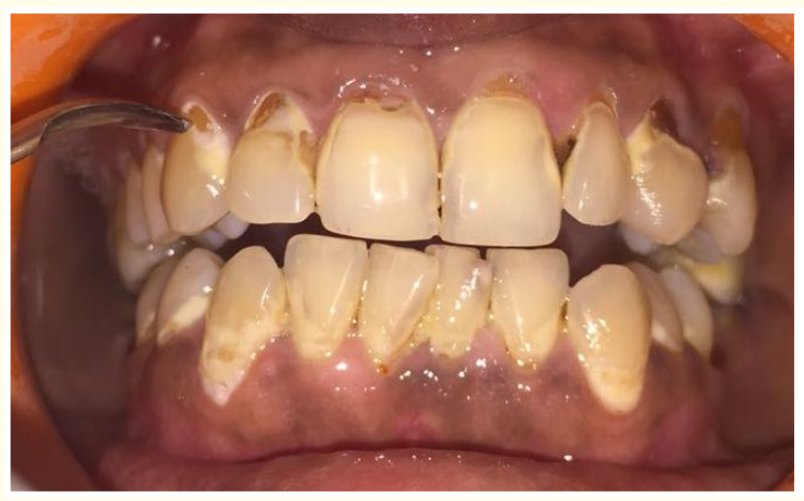

Figure 2

\section{Discussion}

Amelogenesis imperfect can have varied clinical appearances. It can be a mild hypomaturation type with a yellowish discoloration and radiographically as a 'snow capped enamel' appearance. In the hypoplastic type, poor mineralisation at deeper portions while normal mineralisation on surface can be appreciated. Mottled enamel can be appreciated in the hypomaturation type while softer enamel in a hypocalcified type.

\section{Conclusion}

Non-surgical therapy must be carefully done in patients with enamel hypoplasia with the risk of flaking out of most of the enamel and exposing the patient for risk of severe hypersensitivity in future.

\section{Bibliography}

1. Via WF. "Enamel defects induced by trauma during tooth formation”. Oral Surgery, Oral Medicine, Oral Pathology 25 (1968): 49-54.

2. Lv P and Gao XJ. "Phenotype analysis and the molecular mechanism of enamel hypoplasia”. Beijing Da Xue Xue Bao. 41 (2009): 121-123. 
3. Rajendran R and Sundaram S editors. In: Shafer's Textbook of Oral Pathology. 7th ed. India: Elsevier. Developmental disturbances of oral and para oral structures (2012): 49-55.

4. Kumar G. 13th ed. India: Elsevier. Orban's Oral Histology and Embryology (2011): 72-87.

5. Nelson S., et al. "Dental caries and enamel defects in very low birth weight adolescents". Caries Research 44 (2010): 509518.

6. Nikiforuk G and Fraser D. "The etiology of enamel hypoplasia: A unifying concept”. Journal of Pediatrics 98 (1981): 888-893.

7. Gomes AC., et al. "Developmental disturbance of an unerupted permanent incisor due to trauma to its predecessor". Journal of the Canadian Dental Association 76 (2010): a57.

8. Fagrell TG., et al. "Aetiology of severe demarcated enamel opacities - An evaluation based on prospective medical and social data from 17,000 children". Swedish Dental Journal 35 (2011): 57-67.

9. Sapir S and Shapira J. "Clinical solutions for developmental defects of enamel and dentin in children". Pediatric Dentistry 29 (2007): 330-336.

10. Bhushan BA., et al. "Esthetic and endosurgical management of Turner's hypoplasia a sequlae of trauma to developing tooth germ". Journal of Indian Society of Pedodontics and Preventive Dentistry 26 (2008): S121-124.

\section{Volume 3 Issue 8 August 2019}

(C) All rights are reserved by Apeksha Ghai., et al. 